\title{
Automatic Real-Time XRII Local Distortion Correction Method for Digital Linear Tomography
}

\author{
Christian Forlani and Giancarlo Ferrigno \\ Dipartimento di Bioingegneria, Politecnico di Milano, Piazza Leonardo da Vinci 32, 20133 \\ Milano (Italia) \\ \{forlani, ferrigno\}@biomed.polimi.it \\ http: //www.biomed.polimi.it
}

\begin{abstract}
An x-ray image intensifier (XRII) has many applications in diagnostic imaging, especially in real time. Unfortunately the inherent and external distortions (pincushion, S-distortion and local distortion) hinder any quantitative analysis of an image. In this paper an automatic real-time local distortion correction method for improving the quality of digital linear tomography images is presented. Using a digital signal processing (DSP), this method can work for an image up to $1 \mathrm{~K} \times 1 \mathrm{Kx} 12$ bit at $30 \mathrm{fps}$. A local correction method has been used because it allows distortions such as those caused by poor axial alignment between the X-Ray cone beam and the XRII input surface and local distortions to be resolved that are generally neglected by global methods.
\end{abstract}

\section{Introduction}

Linear tomography is a helpful diagnostic exam that permits the acquisition of an $\mathrm{x}$ ray relative to any plane, parallel to the $\mathrm{x}$-ray table. However, inherent and external disturbances on the acquisition system chain cause several distortions when images are acquired by means of a digital system (XRII coupled with a CCD camera) [1][2]. The aim of this work is the development of an automatic real-time method for the correction of geometric distortions in digital linear tomography applications. A local correction algorithm has been used because it guarantees an efficient distortion correction only where needed. Geometric distortions considered and corrected here are: S-distortion, pincushion distortion, asymmetrical and misaligned axes, and local distortion.

\section{Material and Methods}

The method needs a preliminary calibration setup based on the acquisition of a radio opaque square metal grid. For this work a $5 \times 5 \mathrm{~mm}^{2}$ cell size and a $2 \mathrm{~mm}$ metal wire thickness have been chosen, but no restrictions are imposed by the method. The acquisition-system consisted of 16" XRII tube coupled with a $1 \mathrm{Kx} 1 \mathrm{Kx} 12 \mathrm{bit} \mathrm{CCD}$ mounted on GMM Opera X-Ray table. The KV and mAs have been selected for 
guaranteeing an optimal contrast between grid and background with no image saturation. The DSP used for real-time application was a Matrox Genesis@ PCI board working under Windows NT O.S..

\section{Automatic Grid Detection and Classification}

Unlike other wire intersection point detection methods [3], this method uses as control points, the grid cells' Centers Of Gravity (COG) calculated from the acquired grid image by using a hardware blob analysis technique. This results in a reduction in the sensitivity to noise during control point set up. In order to exclude imperfect information about image cells across a radiographic circle, a preliminary circular region threshold has been applied. After thresholding, the method begins to classify the principal distorted axes, starting from the central COG. Cell classification has been obtained by an iterative procedure which is able to find the COG closest to a classified one along a desired direction, starting from a classified one. Nearest neighborhood COG can be found even if its position is shifted up to half a side of an expected square. The minimum distorted axis length is used to define the maximal squared region size where correction is applied. Once the principal axes are calculated, their information are used to classify all the COGs belonging to each quadrant region included in the square-shaped regions. Due to the presence of the radiographic circle and in order to guarantee the maximal dimension of the correction area, external circle COG cells are estimated by means of bilinear interpolation. Classified and estimated COG coordinates are ordered into a matrix. The central cell's area and COG are used to estimate the default cell and wire size parameters useful for the correction algorithm.

\section{Correction Algorithm}

The correction algorithm uses two matrixes of COG points in order to create two biDimensional Look Up Tables (2D LUT) used by a DSP to perform a real-time distortion correction. The first matrix (DM) containing distorted COG coordinates is obtained from the above described grid classification procedure. The second matrix (CM) is filled with corresponding corrected COG coordinates calculated using the default parameters (default cell and wire sizes), starting from the central distorted COG position. Each polygon formed by four adjacent elements (COG points) contained in the first matrix defines each single distorted local area. In the second matrix, the corresponding corrected square local area is defined by four elements located in the same matrix coordinates (see black points in Fig.1.). Each local area is corrected by using a $2 \mathrm{D}$ polynomial function characterized by 20 parameters. In order to calculate all parameters, sixteen COG points have been used: four concerning the local area and twelve belonging to the boundary of the square region. The COG information of the boundaries has been used to improve image continuity across neighboring local areas. The parameters obtained for each local area are used to create a 2D LUT that is used by the DSP image's real-time correction. 

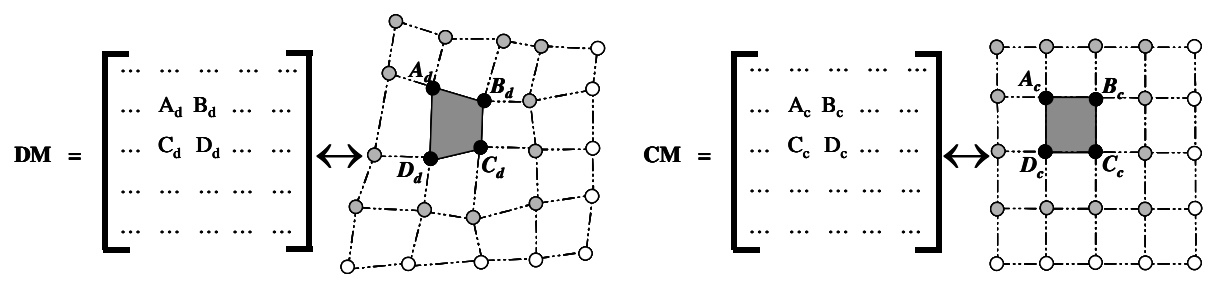

Fig. 1. Generic distorted local area $\left(A_{d} B_{d} C_{d} D_{d}\right)$ and corresponding corrected local area $\left(\mathrm{A}_{c} \mathrm{~B}_{c} \mathrm{C}_{c} \mathrm{D}_{c}\right)$, used to correct distortion. Local area is delimited by four COG (black points), while boundary region (gray COG points) is used to guarantee continuity across neighboring local areas.

\section{Linear Tomography Correction}

The acquisition procedure, when traditional films are used, consists of continuous image integration during generator tilting ( $₫ 20$ degrees) and film shifting. The result is an x-ray image focused on a plane that is parallel to the table at the center of rotation. When digital equipment is used, the film is replaced by an XRII and a CCD and continuous integration is replaced by a discrete image integration. As noted before, due to XRII disturbance, each frame's results are affected by both typical XRII distortions as well as by angular distortions (see Fig.2a).

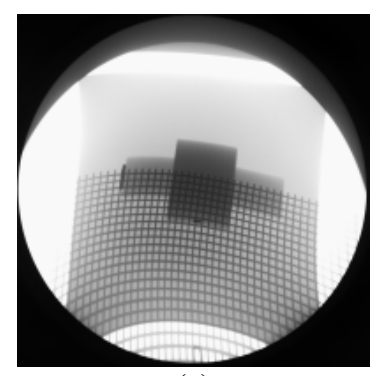

(a)

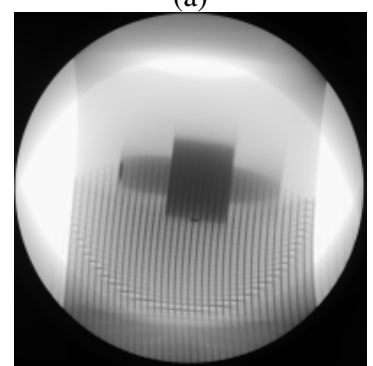

(c)

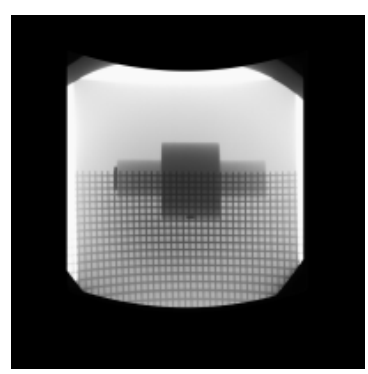

(b)

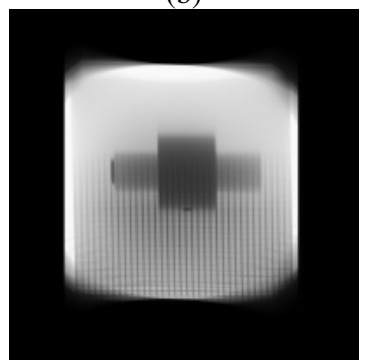

(d)

Fig. 2. Single frame distorted (a) and corrected (b). Linear Tomography distorted (c) and corrected (d). 
As a consequence, the linear tomography obtained by frames integration, with no correction, is highly smoothed and distorted (see Fig.2c). Ideally, in order to obtain a correct digital linear tomography image, each frame should be corrected by means of an angularly dependent global LUT before integration, but that would be time and computationally intensive. The method used and presented in this paper is based on the hypothesis that S-distortion and pincushion distortion are invariant during generator tilting. With this assumption, only one 2D global LUT was calculated when an X-ray cone beam axis was perpendicular to the XRII input surface. The residual angular warping is corrected by fast global cosine correction method. In Fig.2b a corrected single frame, where the distortion and warping are substantially reduced, is shown. Comparing Fig.2c and Fig.2d, it is possible to note how the XRII disturbance, especially the S-distortion and pincushion distortions, deform and rotate the image reducing its quality.

\section{Conclusion}

An automatic local method to correct XRII image distortion in order to increase digital linear tomography image quality has been developed. Neither a grid cell size limit, or distortion information are needed in order to create 2D global LUT, with the exception of neighbor shift limit condition. Local correction allows the reduction of local distortions without significant modification to the rest of image. Image continuity has been guaranteed by using information about neighbor bounding cells. In simulated and real image tests, the residual distance mean errors were about $2.5 \%$ of the initial ones, corresponding to about $0.38 \mathrm{~mm}$. It is expected that the use of a higher grid cell number can further reduce the residual error. The method is based on the hypothesis that the $\mathrm{S}$ and pincushion distortions are invariant during linear tomography acquisition. By using a DSP after set up procedure, this method guarantees a real time operation with resolution up to $1024 \times 1024 \times 12$ bits. The method is completely self-setting, meaning that it can be directly hardware implemented as part of CCD camera. Its notable generality also permits its use in a wide range of image processing applications, not only in digital x-ray.

\section{References}

1. J.M. Boone, "Analysis and correction of imperfections in the image intensifier-TV-digitizer imaging chain” Med. Phys. 18(2), 236-242(1991).

2. S. Rudin, D.R. Bednarek and R. Wong, "Accurate characterization of image intensifier distortion", Med Phys. 18(6), 1145-1151(1991).

3. D.A. Reimann and M.J. Flynn, "Automated Distortion Correction of X-ray Image Intensifier Imager" Nuclear Science Symposium and Medical Imaging Conference, 2, 13391341(1992). 